\title{
A Strategy for Improving the Quality of Ghost Imaging
}

\author{
Xin Liu, ${ }^{1,2,3,4}$ Su Bo $\left(\mathbb{D},{ }^{1,2,3,4}\right.$ Zhiyuan Ye, ${ }^{4}$ Panghe Qiu, ${ }^{4}$ and Jing Ding ${ }^{4}$ \\ ${ }^{1}$ Key Laboratory of Terahertz Optoelectronics, Ministry of Education, Beijing 100048, China \\ ${ }^{2}$ Beijing Key Laboratory for Terahertz Spectroscopy and Imaging, Beijing 100048, China \\ ${ }^{3}$ Beijing Advanced Innovation Centre for Imaging Theory and Technology, Beijing 100048, China \\ ${ }^{4}$ Department of Physics, Capital Normal University, Beijing 100048, China \\ Correspondence should be addressed to Su Bo; subo75@cnu.edu.cn
}

Received 13 January 2020; Revised 12 March 2020; Accepted 19 March 2020; Published 9 April 2020

Academic Editor: Adrian Podoleanu

Copyright (C) 2020 Xin Liu et al. This is an open access article distributed under the Creative Commons Attribution License, which permits unrestricted use, distribution, and reproduction in any medium, provided the original work is properly cited.

\begin{abstract}
A new method is proposed to improve the signal-to-noise ratio (SNR) of regions of interest (ROIs) in a ghost imaging (GI) system with uneven speckle illumination. The imaging results in a GI system can be distorted when there is an uneven distribution of light. In this study, three thin-film polarizers are used to create illumination patterns in uneven light intensity distribution. In particular, the polarizer set is loaded on the object arm only, that is, the original uniformly distributed light field is still acquired by the reference arm. This small change in the light path eliminates the distortion caused by uneven illumination while increasing the SNR of the ROI. This strategy has been confirmed in principle and through simulation and experiments.
\end{abstract}

\section{Introduction}

Ghost imaging (GI) [1-6], also known as correlated imaging, can recover the information of an object utilizing a detector without spatial resolution. The classic GI system typically consists of two arms; one (the reference arm) directly collects the spatial distribution of the light source with an area array detector, and the other (the object arm) collects the intensity information of the object through a bucket detector. This information, acquired synchronously on the two arms, can recover the image of the target through a correlation operation.

However, traditional schemes cannot reconstruct images to a very high quality, even when there are a large number of samples, and such an imaging system requires many hours of sampling time prior to the acquisition of real-time imaging of dynamic scenes. In recent years, many optimized schemes [7-14] have been used to improve this situation, such as computational [7], differential [8], normalized [9], compressive sensing computational [10, 11], sinusoidal [12], high-order [13], and pseudoinverse [14] GI. One of the significant advantages of GI over traditional imaging systems is the ability to recover images in low light conditions $[15,16]$ or with large disturbances. [17-20].

In conventional GI systems, laser light is applied to the rotating ground glass to generate thermal light. With the rotation of the frosted glass, the thermal light can be seen as a set of time-varied illumination patterns of Gaussian distribution. The illumination mode is, of course, closely related to the imaging quality $[12,21-23]$, and uneven distribution of light intensity in the illumination modes will redistribute the signal-to-noise ratio (SNR) [24] in addition to the distortion of the image. Consequently, actively generated uneven light fields can improve the SNR of local ROIs. In this study, three thin-film polarizers were used to create illumination patterns of the uneven light intensity distribution. The polarizer set was loaded on the object arm only, that is, the original uniformly distributed light field was still acquired by the reference arm. This small change in the light path contributed to the elimination of the distortion caused by uneven illumination (no additional postdigital processing was required) while increasing the SNR of the region of interest (ROI). This strategy has been confirmed in principle and through simulation and experiments. 


\section{Modulation of an Uneven Light Field}

As shown in Figure 1, in the optical path, we used two freely rotatable angle film polarizers, $P_{1}$ and $P_{3}$, and a ringshaped film polarizer $P_{2}$ loaded with a two-dimensional translation stage, to modulate the light field and produce an uneven speckle pattern. It should be noted that although the aperture can achieve infinite SNR, it cannot provide a condition for an uneven spot. To unify the relative yaw angles of the three thin-film polarizers, the original angle of the intermediate annular film polarizer is $0^{\circ}$, and the angle values obtained by the clockwise rotation of the two front and back film polarizers are $\alpha$ and $\beta$, respectively.

The initial illuminance is represented by $I_{0}$. After passing through three thin-film polarizers, the illuminates of the middle circular area and outer annular area are represented by $I_{2}$ and $I_{1}$, respectively. According to Malus's law, the following equation can be obtained:

$$
r=\frac{I_{2}}{I_{1}}=k(\alpha, \beta)(1+\tan \alpha \quad \tan \beta),
$$

where the value of $r$ represents the ratio of the illuminance of the circular area to the illuminance of the outer ring area. Furthermore, the value of $k(\alpha, \beta)$ indicates the loss factor due to the absorption of light by the polarizer in the actual case.

As shown in Figure 2, a variety of speckle patterns were obtained from the three film polarizers. Figure 3 shows the theoretical and practical relationship between the value of $\log _{10}(r)$ and the third polarizer rotation angle $\beta$. It can be seen that the value of $r$ is theoretically broad and not precise. In fact, the exact value of $r$ is of little significance in specific experiments and reflects a qualitative relationship. We fixed the value of $\alpha$ to $30^{\circ}$, that is, the first film polarizer and the second ring polarizer remained unchanged so that the third film polarizer could be adjusted to achieve different values of $r$.

\section{GI System with the Polarizer Group}

The setup of Experiment A is shown in Figure 4. The red light generated by the $\mathrm{He}-\mathrm{Ne}$ laser (Peking University Physics Department Factory, JD2B-0506013) became linearly polarized light through polarizer P1. The rotating ground glass, which was controlled by a stepper motor, modulated the laser into thermal light. The thermal light first passed through the pinhole, and then the beam splitter, to become two paths of light. On one of the paths, the speckle pattern was directly collected by a charge-coupled device (CCD) (Daheng MER-031-U3M) at position M1. On the other path, the beam passed through lens $L 1(f=50 \mathrm{~mm})$ and annular film polarizer $P 2$ to reach the object plane, where $M 1$ and $M 2$ were equidistant from the beam splitter, and the position of M2 satisfied the imaging relationship with the object plane. After passing through the object, the beam passed through lens $L 2(f=30 \mathrm{~mm})$ and polarizer $P 3$ and was collected by an amplified photodetector (APD) (Thorlabs PDA100A2), which converted the optical signal into an electrical signal and amplified it. Finally, the electrical signal was converted into a digital signal by the data acquisition (DAQ) card (NI PCI-6220) and transmitted to the computer. In data processing, the speckle patterns and simultaneously acquired intensity values were used to recover the image.

To facilitate the mathematical derivation, we used matrix operations to describe the physical processes of the GI. We used the $N$-dimensional row vector $\mathbf{O}(1 \times N)$ to represent the target for imaging, where the value of $N$ represents the number of pixels of the recovered object. Traditional GI requires high quantities of illumination patterns or speckle patterns to be effective. Here, we used the measurement matrix $\mathbf{S}(M \times N)$ to represent different speckle patterns or illumination patterns $(M)$. These $M$ illumination patterns were projected onto the object, their reflected or transmitted light was collected by a converging lens, and a bucket detector was used to collect the light intensity. This physical process can be approximated by the following equation:

$$
\mathbf{b}=\mathbf{O S}
$$

where vector $\mathbf{b}(1 \times M)$ represents the relative intensity values detected by the single-pixel detector.

In a GI system, it is common to use the following equation to recover the image of the object:

$$
\widehat{O}=(\mathbf{b}-\langle\mathbf{b}\rangle) \mathbf{S}^{T},
$$

where $\widehat{O}$ represents the recovery solution after imaging of $\mathbf{O}$, $\langle\mathbf{b}\rangle$ represents the mean of all elements in vector $\mathbf{b}$, and ${ }^{T}$ represents the transpose of the matrix.

To better illustrate the applications of the ROI, we divide the vector $\mathbf{O}$ into two parts, $\mathbf{O}_{1}\left(1 \times n_{1}\right)$ and $\mathbf{O}_{2}\left(1 \times n_{2}\right)$, where $n_{1}+n_{2}=N$ and $\mathbf{O}_{1}$ represents the object corresponding to the intermediate ROI, and $\mathbf{O}_{2}$ represents the object corresponding to other regions, as shown in the following equation:

$$
\mathbf{O}=\left[\mathbf{O}_{1}, \mathbf{O}_{2}\right]
$$

Accordingly, $\mathbf{S}$ can also be divided into two parts, $\mathbf{S}_{1}\left(n_{1} \times M\right)$ and as shown in the following equation:

$$
\mathbf{S}=\left[\begin{array}{l}
\mathbf{S}_{1} \\
\mathbf{S}_{2}
\end{array}\right]
$$

Due to the modulation of the three polarizers, the ratio of the illuminance in the inner circular area to the outer circular area is $r$, so equation (5) can be written as follows:

$$
\mathbf{S}^{\prime}=\left[\begin{array}{c}
r \mathbf{S}_{1} \\
\mathbf{S}_{2}
\end{array}\right]
$$

Analogous to equation (2), the following equation can be obtained:

$$
\mathbf{b}^{\prime}=\mathbf{O S}^{\prime}
$$

Ignoring the constant term $\left\langle\mathbf{b}^{\prime}\right\rangle$ and substituting equation (7) into equation (3) yields 


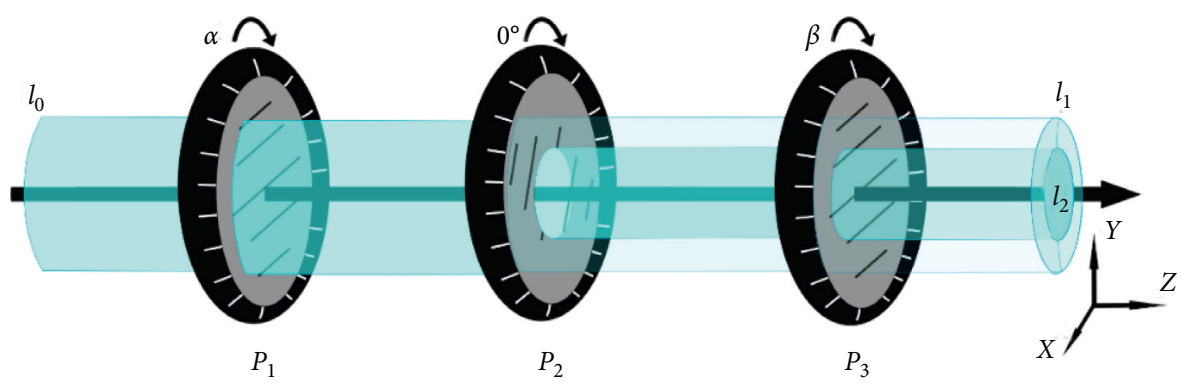

FIgURE 1: Schematic diagram of the three thin-film polarizers.

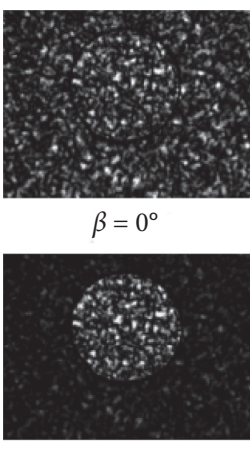

$\beta=60^{\circ}$

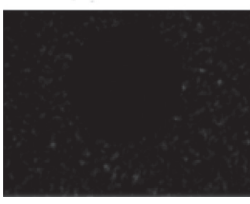

$\beta=120^{\circ}$

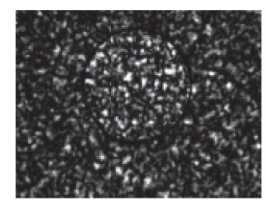

$\beta=10^{\circ}$

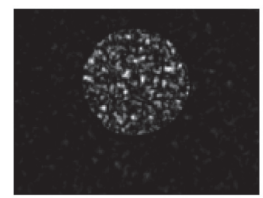

$\beta=70^{\circ}$

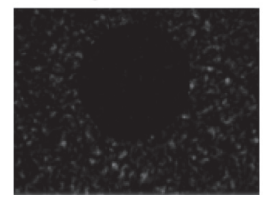

$\beta=130^{\circ}$

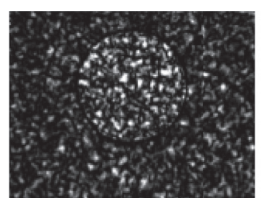

$\beta=20^{\circ}$

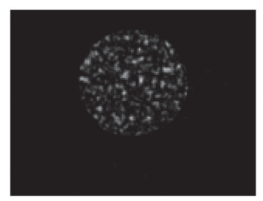

$\beta=80^{\circ}$

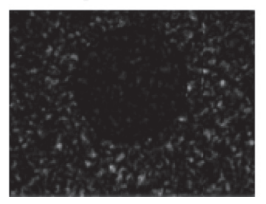

$\beta=140^{\circ}$

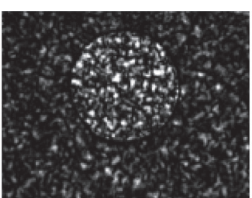

$\beta=30^{\circ}$

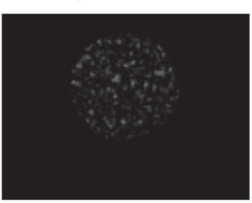

$\beta=90^{\circ}$

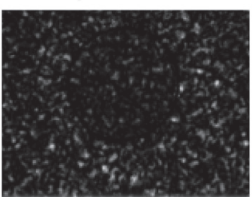

$\beta=150^{\circ}$

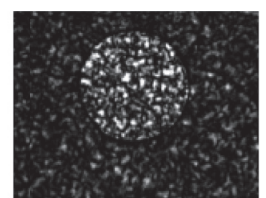

$\beta=40^{\circ}$

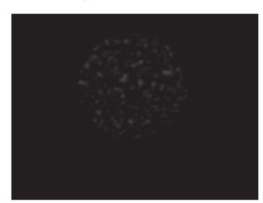

$\beta=100^{\circ}$

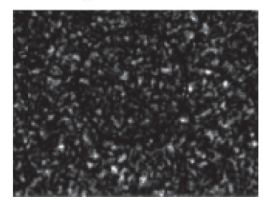

$\beta=160^{\circ}$

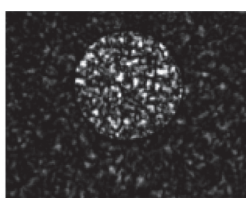

$\beta=50^{\circ}$

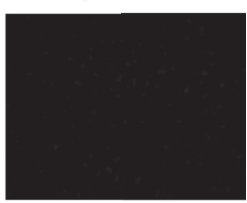

$\beta=110^{\circ}$

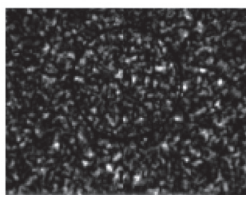

$\beta=170^{\circ}$

Figure 2: Set of speckle patterns obtained after three film polarizers.

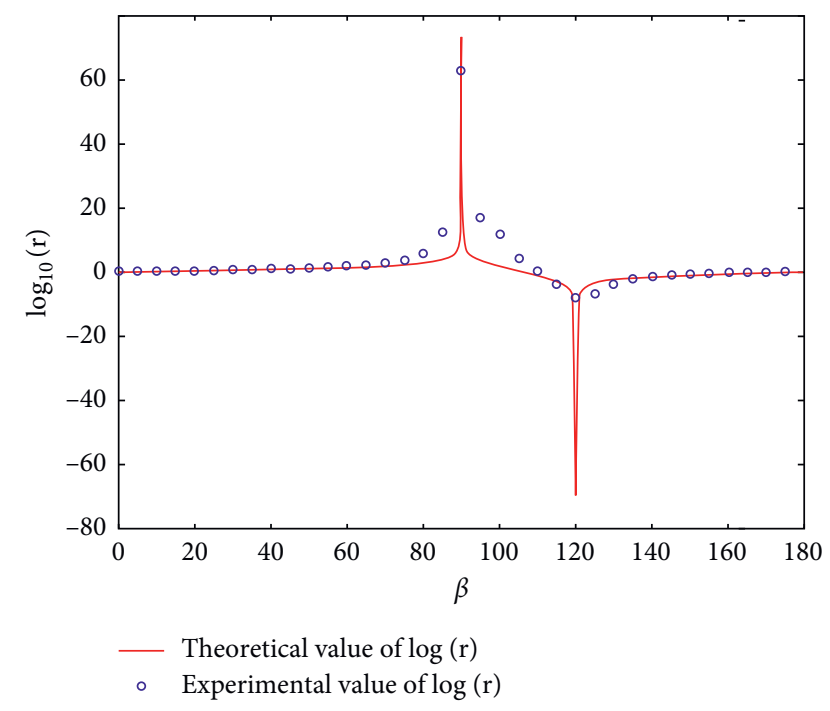

FIgURE 3: Theoretical and practical relationship between the value of $\log _{10}(r)$ and third polarizer rotation angle $\beta$ when $\alpha=30^{\circ}$. Their error is within an acceptable range, and the error is mainly due to the nonlinear coefficient $k(\alpha, \beta)$ and the limited dynamic range of the charge-coupled device (CCD).

$$
\widehat{O}^{\prime}=\left[\begin{array}{c}
\widehat{O}_{1}^{\prime} \\
\widehat{O}_{2}^{\prime}
\end{array}\right]=\mathbf{O S}^{\prime} \mathbf{S}^{T}=\left[\begin{array}{c}
r \mathbf{O}_{1} \mathbf{S}_{1} \mathbf{S}_{2}^{T}+\mathbf{O}_{2} \mathbf{S}_{2} \mathbf{S}_{1}^{T} \\
\mathbf{O}_{2} \mathbf{S}_{2} \mathbf{S}_{2}^{T}+r \mathbf{O}_{1} \mathbf{S}_{1} \mathbf{S}_{2}^{T}
\end{array}\right] .
$$

Equation (8) shows $\mathbf{O}_{\mathbf{1}}$ is in a competitive relationship with $\mathbf{O}_{2}$. Though we cannot precisely control $\mathbf{S}$ in traditional GI, we can adjust the value of $r$ by adjusting the angle of the polarizer in our system. In this way, $\mathbf{S}_{1} \mathbf{S}_{1}^{T}, \mathbf{S}_{2} \mathbf{S}_{1}^{T}, \mathbf{S}_{2} \mathbf{S}_{2}^{T}$, and $\mathbf{S}_{1} \mathbf{S}_{2}^{T}$ cannot be artificially controlled. For $\mathbf{O}_{1}, \mathbf{O}_{2} \mathbf{S}_{2} \mathbf{S}_{1}^{T}$ is an inevitable background noise term, as is $\mathbf{O}_{2}$. Hence, $r \mathbf{O}_{1} S_{1} \mathbf{S}_{2}^{T}$ determines the imaging quality of $\mathbf{O}_{\mathbf{1}}$ under the condition that the background noise term cannot be changed. Under the initial conditions, the value of $r$ is 1 , and its physical meaning is that the polarizer system does not uniformly modulate the speckle pattern. When we rotate the polarizer to make the value of $r$ greater than 1, the irrelevant background noise term of $\mathbf{O}_{\mathbf{1}}$ is fully suppressed and the irrelevant background noise term of $\mathbf{O}_{2}$ is amplified, and vice versa.

If an uneven light field is also loaded into the reference arm, the following equation can be obtained:

$$
\widehat{O}=\left[\begin{array}{c}
\widehat{O}_{1} \\
\widehat{O}_{2}
\end{array}\right]=\mathbf{O S}^{\prime} \mathbf{S}^{\prime T}=\left[\begin{array}{c}
r^{2} \mathbf{O}_{1} \mathbf{S}_{1} \mathbf{S}_{2}^{T}+r \mathbf{O}_{2} \mathbf{S}_{2} \mathbf{S}_{1}^{T} \\
\mathbf{O}_{2} \mathbf{S}_{2} \mathbf{S}_{2}^{T}+r \mathbf{O}_{1} \mathbf{S}_{1} \mathbf{S}_{2}^{T}
\end{array}\right]
$$




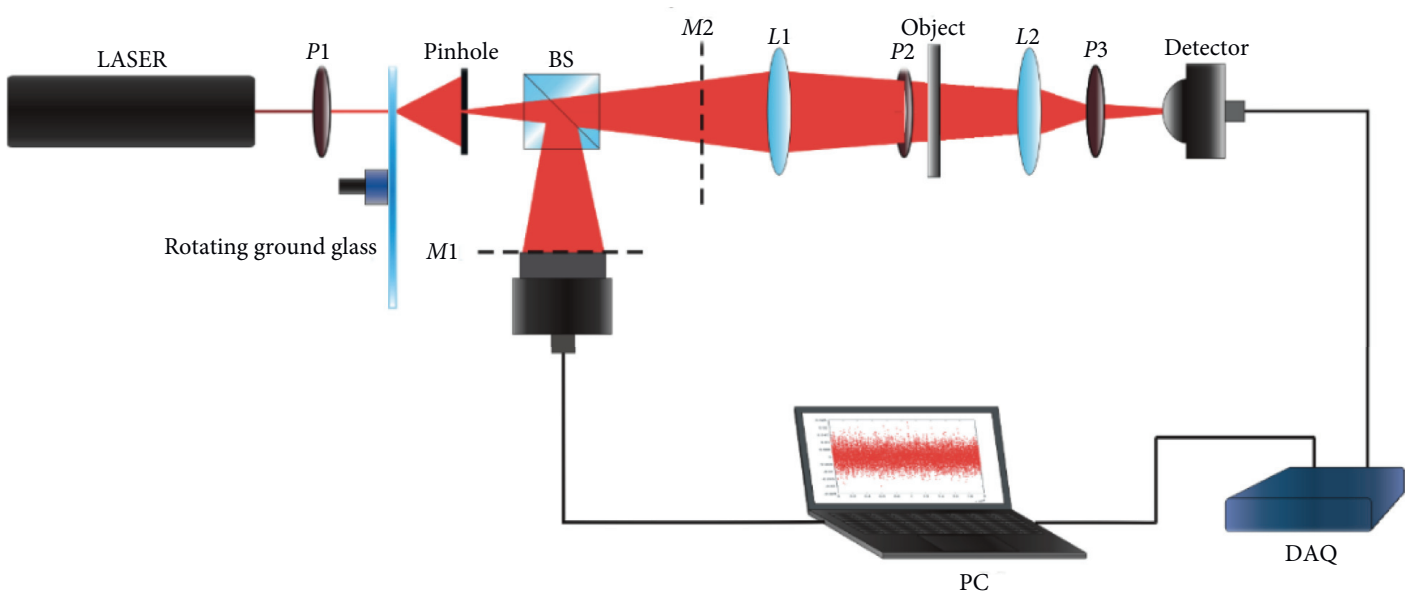

Figure 4: Schematic diagram of the experimental setup.

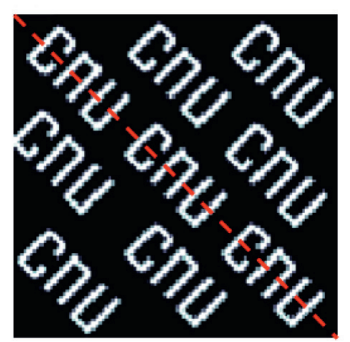

(a)

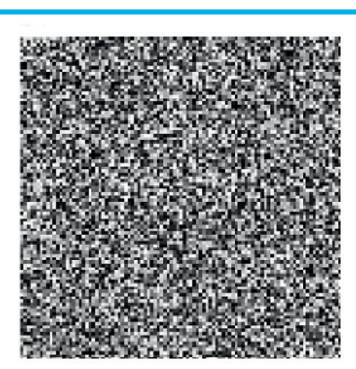

(b)

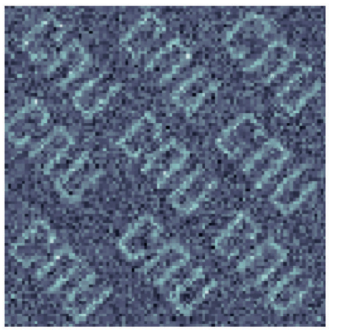

(c)

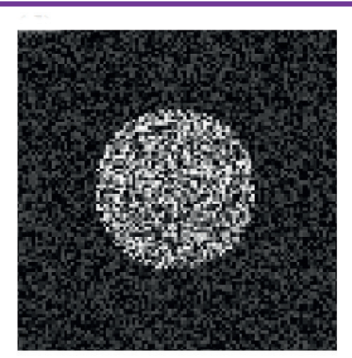

(d)

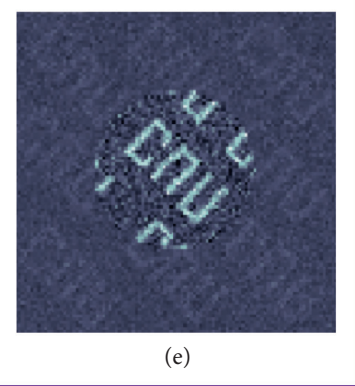

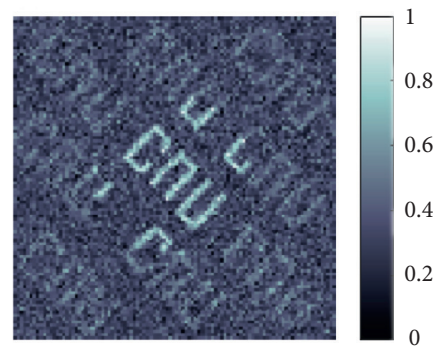

(f)

Figure 5: Simulation results of the "cnu" image. (a) Original image. (b) Speckle pattern with uniform light intensity distribution. (c) Simulation result based on (b) (10,000 measurements). (d) Speckle pattern with uneven light intensity distribution ( $r=3$ ). (e) Simulation result based on (d) (10,000 measurements). (f) Simulation result obtained by the proposed method, that is, the uneven light field is only loaded in the object arm.

By comparing equations (8) and (9), it can be seen that it will result in a significant linear distortion to the imaging when the uneven light field exists simultaneously within the two arms in the GI system. This linear distortion does not further increase the SNR of the ROI because it amplifies the signal and scales the noise equally. When the polarization group is applied to the object arm of the GI system, the multiplicative distortion is eliminated, improving the SNR of the ROI.

\section{Simulation and Experimental Results}

A set of simulation results is given below. In the simulation, we used a mask pattern of "cnu" of size $100 \times 100 \mathrm{~mm}^{2}$ as the imaging target. After collecting 10,000 images and using equation (3), the image of the target was recovered, as shown in Figure 5. Figure 5(a) shows images waiting to be analyzed. Figure 5(b) shows the computer-simulated speckle pattern of a Gaussian distribution. Figure 5(c) shows the imaging result using the original speckle patterns. Figure 5(d) shows the speckle pattern in which the intensity distribution is uneven, with the intensity of the ROI at three times that of other regions, that is, $r=3$. Figure $5(\mathrm{e})$ shows the imaging result using an uneven light field in both arms. Figure 5(f) shows the imaging result obtained by the proposed method, that is, the uneven light field is loaded only into the object arm. It can be seen from the results that the visibility and SNR of the ROI are significantly higher than the original imaging results after the introduction of the uneven light field. It is inevitable, however, that the visibility and SNR of 


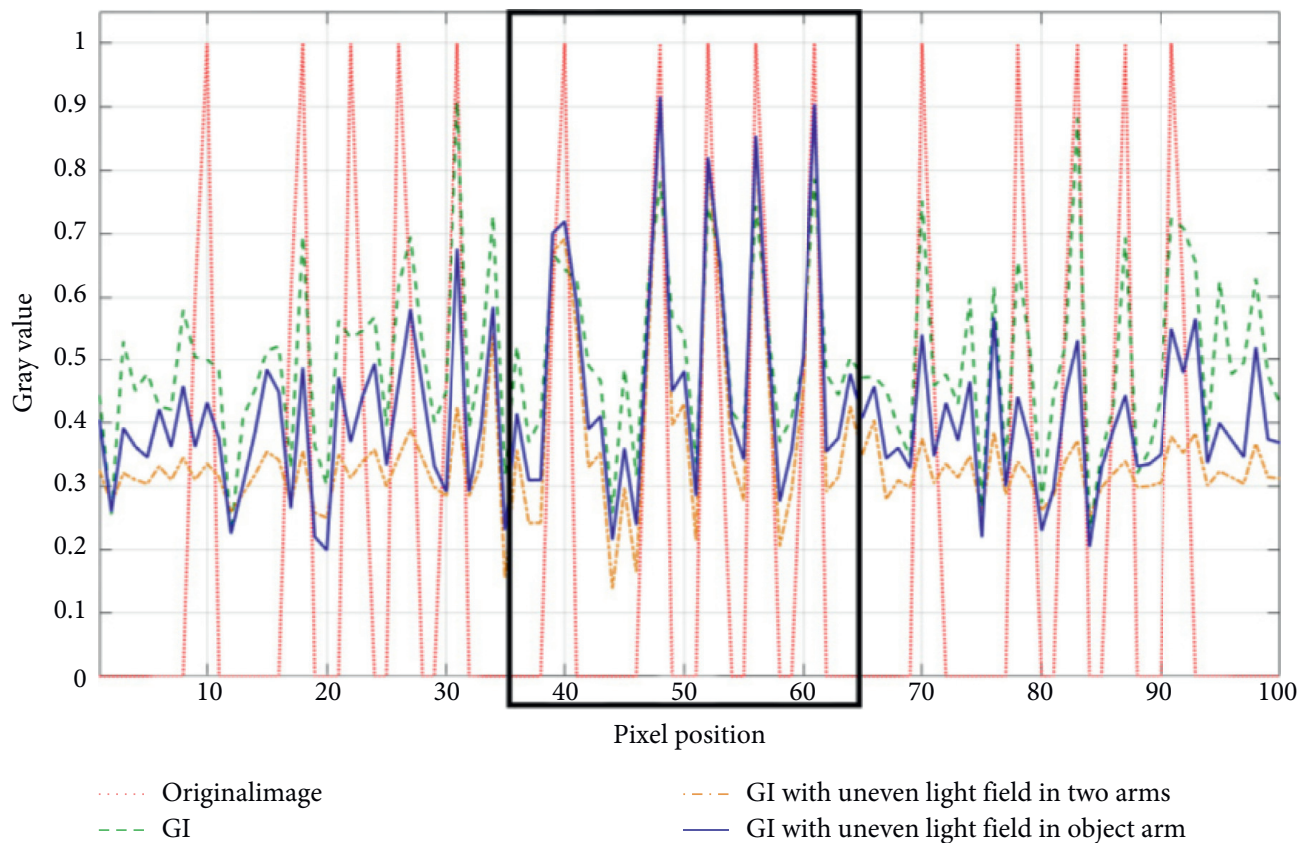

Figure 6: Comparison of the simulation results of Figure 5. Gray values of a target region for Figures. 5(a), 5(c), 5(e), and 5(f) as a function of pixel position, the dashed line indicates the gray value distribution of the imaging target. Inside the black wireframe is the ROI.

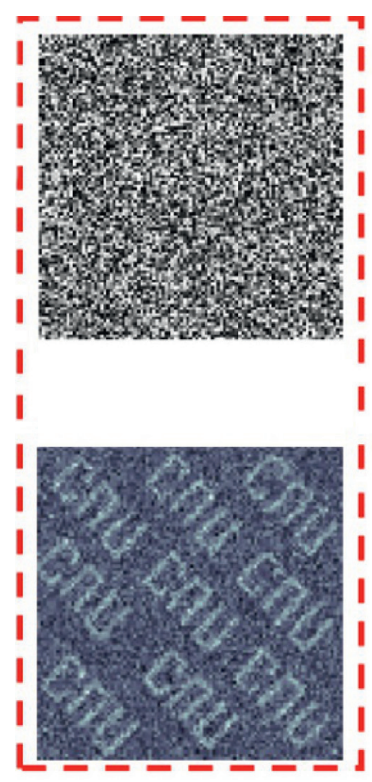

(a)

I
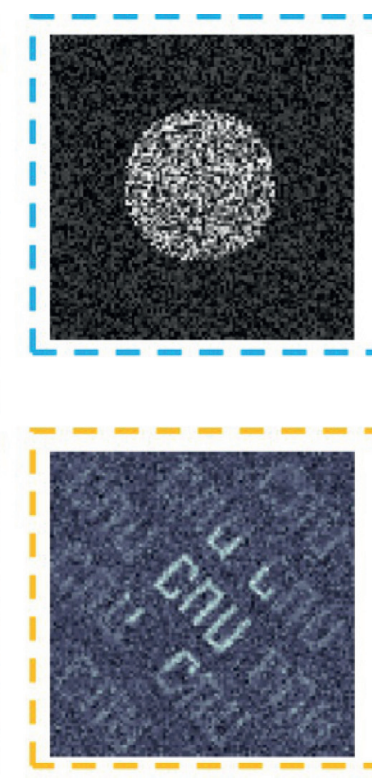
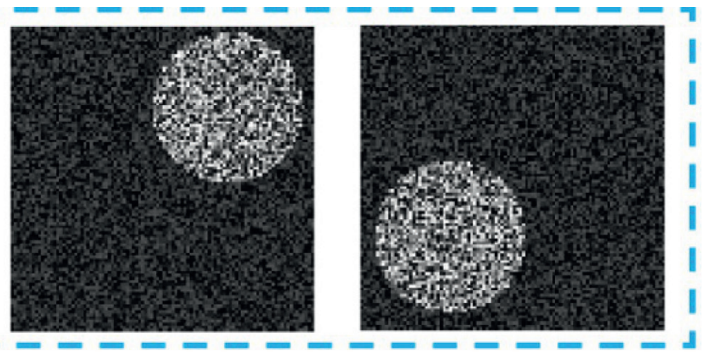

(b)
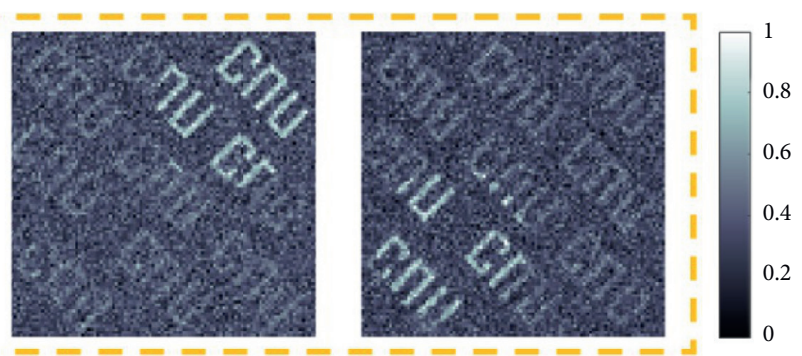

(c)

Figure 7: A set of simulation results of different ROIs using the proposed method. (a) The original speckle patterns and the imaging results $(10,000$ measurements). (b) The speckle patterns with uneven light intensity in different ROIs. (c) Imaging results (10,000 measurements, $r=3$ ) corresponding to the speckle patterns in (b).

other regions would decrease accordingly under the same number of sampling times, and the data in Figure 6 show that we obtained this result. Figure 6 shows the gray values of the corresponding regions for Figures 5(a), 5(c), 5(e), and $5(f)$ as a function of pixel position, where the dashed line indicates the gray value distribution of the imaging target in Figure 5(a). When comparing Figures 5(e) and 5(f), it can be seen that the light intensity distribution of the imaging result in Figure 5(e) is uneven and there is significant distortion, while Figure 5(f) appears to be quite natural. The simulation results confirm the proposed theory.

Figure 7 shows a set of simulation results of different ROIs using the method proposed. The setting parameters of the simulations are the same as mentioned before, the number of measurements is 10,000 and $r=3$. Comparing the imaging results of figures $7(\mathrm{a})$ and $7(\mathrm{c})$, it is evident that the imaging results with the polarizer system do indeed improve the SNR. In the specific experiment described below, we 


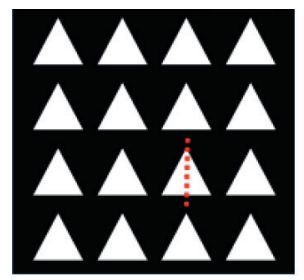

(a)

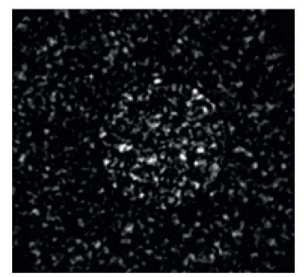

(e) $\beta=30^{\circ} r=1.78$

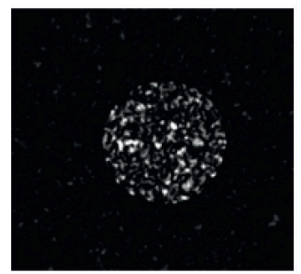

(i) $\beta=66^{\circ} r=5.28$

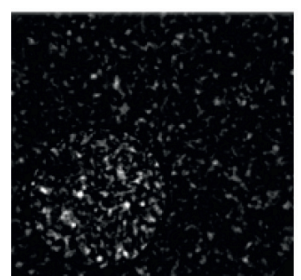

(m) $\beta=30^{\circ} r=1.78$

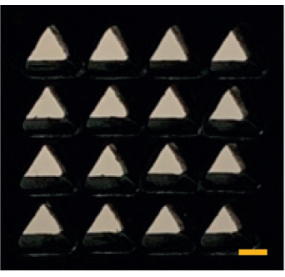

(b)

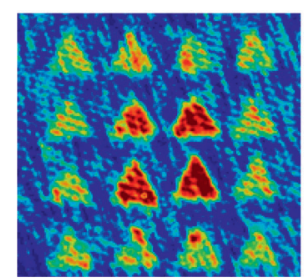

(f)

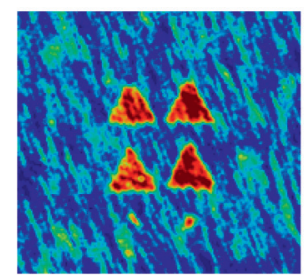

(j)

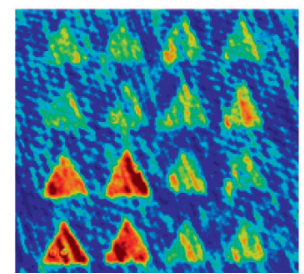

(n)

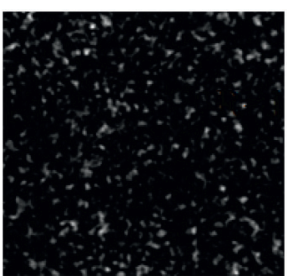

(c)

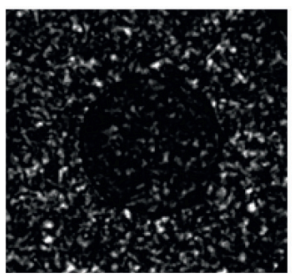

(g) $\beta=150^{\circ} r=0.44$

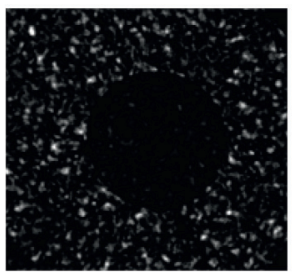

(k) $\beta=138^{\circ} r=2.23$

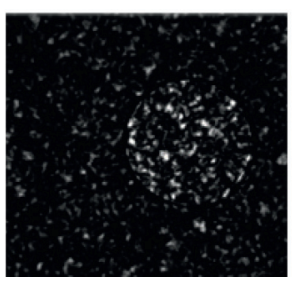

(o) $\beta=30^{\circ} r=1.78$

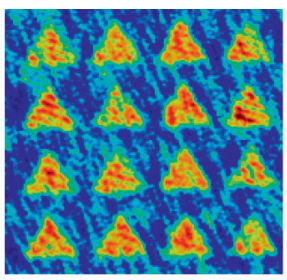

(d)

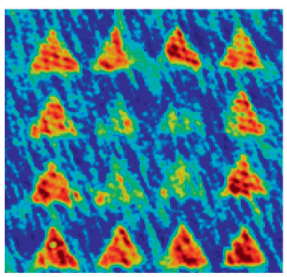

(h)

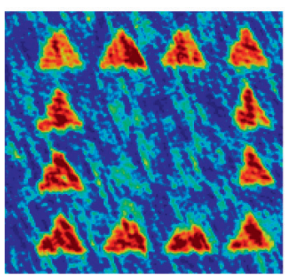

(l)

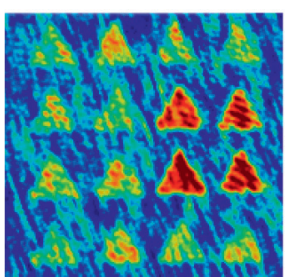

(p)

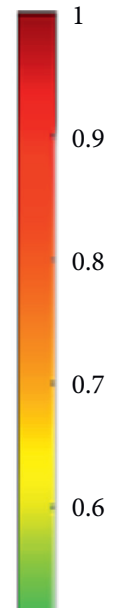

0.5

0.4

0.3

0.2

0.1

FIgURE 8: Experimental result of the periodic projection target. (a) The periodic projection target; (b) image of the object captured by the CCD; (c) the original speckle pattern captured by the CCD; (d) the imaging results of (c); (e), (g), (i), and (k) speckle patterns modulated at different polarization angles in the same ROI; (f), (h), (j), and (l) the corresponding imaging results $(10,000$ measurements); (m) and (o) speckle patterns at the same polarization angles in different ROIs; (n) and (p) the corresponding imaging results (10,000 measurements). The results of this experiment were all subjected to pseudocolor processing. Scale bar $=2 \mathrm{~mm}$.

controlled the spatial position of the circular polarize $P_{2}$ through a two-dimensional translation stage and actively selected the position of the ROI. This ensured a more practical implementation, as, for example, the target could be dynamically sampled to recover the image with high SNR.

As shown in Figures 8(a) and 8(b), a periodically distributed triangular pattern is engraved onto a black acrylic plate, in which the black portion is opaque, and the white portion is transparent. A total of seven comparative experiments on the object were undertaken, visually demonstrating the difference in imaging through the experimental results. The following experiments were performed for 10,000 sampling times, the rotational speed of the ground glass was set at 5,000 steps per revolution, and the acquisition frame rate of the CCD was $2 \mathrm{~Hz}$. The angle between the first polarizer and second annular polarizer $\alpha$ was $30^{\circ}$.

Comparing Figures 8(d), 8(f), and 8(j), as the value of $r$ increased, the SNR and contrast of the ROI increased accordingly. Figures $8(\mathrm{~d}), 8(\mathrm{~h})$, and $8(\mathrm{l})$ show that the SNR and contrast of the ROI decreased correspondingly until the ROI was submerged by the background noise. Compared with figures $8(\mathrm{~d})$ and $8(\mathrm{f})$, it can be seen that the imaging result of the ROI was better when the polarizer system was added. The position of the second polarizer was adjusted by moving the two-dimensional translation stage to obtain the experimental results of Figures 8(n) and 8(p). For a quantitative description and comparison, we selected the area marked with a bold red dashed line in Figure 8(a) as the comparison target. Figure 9 shows the gray values of the corresponding regions for Figures 8(d), 8(f), 8(h), 8(j), and $8(\mathrm{l})$ as a function of pixel position, where the dashed line indicates the gray value distribution of the imaging target. Table 1 introduces the ratio of gray value of different $r$ value and gray value when $r=1$. The table is a supplement to Figure 9. There was no significant intensity distortion in the experimental results. Overall, the imaging results are 


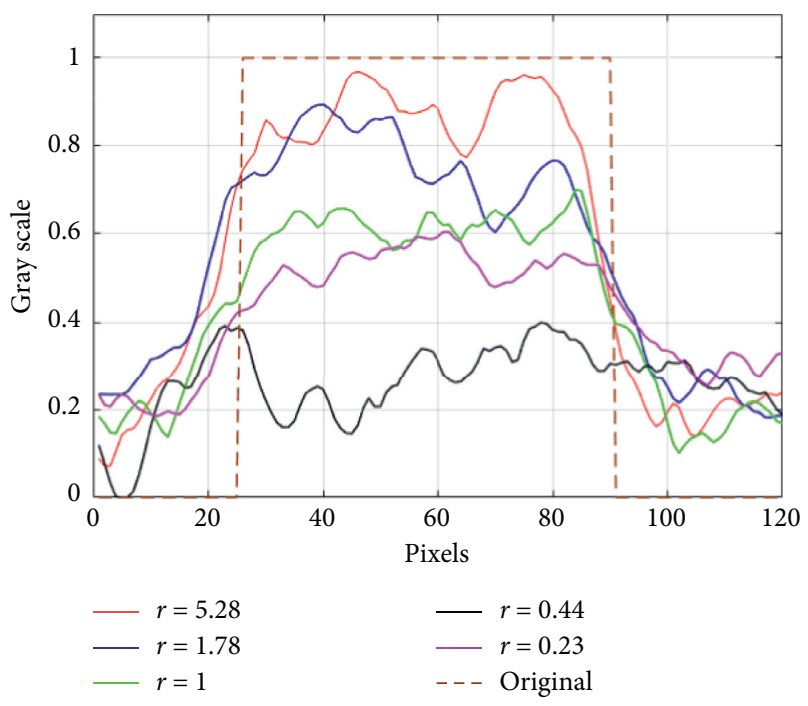

FIGURE 9: Comparison of the experimental results. Gray values of a target region for Figures 8(d), 8(f), 8(h), 8(j), and 8(l) as a function of pixel position, in which the dashed line indicates the gray value distribution of the imaging target.

TABLE 1: The ratio of gray value of different $r$ value and gray value when $r=1$.

\begin{tabular}{lcc}
\hline$r$ & Gray value & Ratio of gray value $(r=1)$ \\
\hline 1 & 0.6 & 1 \\
0.23 & 0.52 & 0.87 \\
0.44 & 0.3 & 0.5 \\
1.78 & 0.78 & 1.3 \\
5.28 & 0.9 & 1.5 \\
\hline
\end{tabular}

comparable to theoretical expectations. Using the proposed system and scheme, we eliminated the influence of uneven light intensity and improved the SNR and contrast of the ROI.

\section{Conclusion}

In conclusion, this study proposed a new method to improve the SNR of ROIs in a GI system with uneven speckle illumination. Three thin polarizers were used to realize the regional reallocation of the SNR, and the polarizer system was loaded into the object arm only. These small changes simultaneously eliminated the distortion caused by uneven illumination while improving the SNR and visibility. Moreover, we flexibly achieved GI with high SNR of multiple different ROIs by adjusting the position of the circular polarizer. This method could also be used when a large number of measurements are required.

\section{Data Availability}

The original data used to support the results of this study have not been provided because we are now conducting another experiment and need to use the original data in this paper.

\section{Conflicts of Interest}

The authors declare that they have no conflicts of interest.

\section{Authors' Contributions}

All authors contributed to the theoretical analysis, calculations, experiment, and preparation of the manuscript.

\section{Acknowledgments}

This work was supported by the National Natural Science Foundation of China (NSFC) (61575131, 61675138, and 61575130) and the Beijing Municipal Commission of Education for financial support (no.SQKM201810028004)). The authors would like to thank 'enago' for providing English touch up.

\section{References}

[1] T. B. Pittman, Y. H. Shih, D. V. Strekalov, and A. V. Sergienko, "Optical imaging by means of two-photon quantum entanglement," Physical Review A, vol. 52, no. 5, pp. R3429-R3432, 1995.

[2] R. S. Bennink, S. J. Bentley, R. W. Boyd et al., Physical Review Letters, vol. 92, p. 033601, 2004.

[3] A. Gatti, E. Brambilla, M. Bache et al., Physical Review Letters, vol. 93, p. 093602, 2004.

[4] G. Scarcelli, V. Berardi, and Y. Shih, Applied Physics Letters, vol. 88, p. R3429, 2006.

[5] L. Basano and P. Ottonello, "Experiment in lensless ghost imaging with thermal light," Applied Physics Letters, vol. 89, no. 9, p. 091109, 2006.

[6] X.-H. Chen, Q. Liu, K.-H. Luo, and L.-A. Wu, "Lensless ghost imaging with true thermal light," Optics Letters, vol. 34, no. 5, p. 695, 2009.

[7] H. J. Shapiro, Physical Review A, vol. 78, p. 061802, 2008.

[8] F. Ferri, D. Magatti, L. A. Lugiato, and A. Gatti, Physical Review Letters, vol. 104, p. 253603, 2010

[9] B. Sun, S. S. Welsh, M. P. Edgar, J. H. Shapiro, and M. J. Padgett, "Normalized ghost imaging," Optics Express, vol. 20, no. 15, p. 16892, 2012.

[10] V. Katkovnik and J. Astola, "Compressive sensing computational ghost imaging," Journal of the Optical Society of America A, vol. 29, no. 8, p. 1556, 2012.

[11] A. Bmann, Marc, and M. Bayer, "Compressive adaptive computational ghost imaging," Scientific Reports, vol. 3, p. 1545, 2013.

[12] S. M. M. Khamoushi, Y. Nosrati, and S. H. Tavassoli, "Sinusoidal ghost imaging," Optics Letters, vol. 40, pp. 3452-3455, 2015.

[13] K. Chan, M. N. O'Sullivan, and R. W. Boyd, "High-Order Thermal Ghost Imaging," IEEE, Piscataway, NJ, USA, 2009.

[14] C. Zhang, S. Guo, J. Cao, J. Guan, and F. Gao, "Object reconstitution using pseudo-inverse for ghost imaging," Optics Express, vol. 22, no. 24, p. 30063, 2014.

[15] P. A. Morris, R. S. Aspden, J. E. C. Bell et al., Nature Communications, vol. 6, p. 5913, 2015.

[16] D. Pelliccia, A. Rack, M. Scheel, C. Valentina, and D. M. Paganin, "Experimental X-ray ghost imaging," Physical Review Letters, vol. 117, p. 113902, 2016.

[17] J. Cheng, "Ghost imaging through turbulent atmosphere," Optics Express, vol. 17, no. 10, p. 7916, 2009. 
[18] P. Ben Dixon, G. Howland, K. W. C. Chan et al., "Quantum ghost imaging through turbulence," Physical Review A, vol. 83, p. 911, 2011.

[19] R. E. Meyers, K. S. Deacon, and Y. Shih, Applied Physics Letters, vol. 98, p. 041801, 2011.

[20] E. Tajahuerce, V. Durán, P. Clemente et al., "Image transmission through dynamic scattering media by single-pixel photodetection," Optics Express, vol. 22, no. 14, p. 16945, 2014.

[21] C. Zhao, W. Gong, M. Chen et al., "Ghost imaging lidar via sparsity constraints," Applied Physics Letters, vol. 101, p. 139, 2012.

[22] L. Wang and S. Zhao, "Fast reconstructed and high-quality ghost imaging with fast Walsh-Hadamard transform," Photonics Research, vol. 4, no. 6, pp. 340, 2016.

[23] C. Yang, C. Wang, J. Guan et al., "Scalar-matrix-structured ghost imaging," Photonics Research, vol. 4, no. 6, p. 281, 2016.

[24] H. Li, J. Shi, and G. Zeng, "Ghost imaging with nonuniform thermal light fields," Journal of the Optical Society of America A, vol. 30, no. 9, p. 1854, 2013. 